\title{
IAC Accounting Data Model: A Better Data Structure For Computerized Accounting Systems
}

Carlos Ferran, (Email: cferran@psu.edu), Penn State, Great Valley Ricardo Salim, (Email: rsalim@cautus.net), Cautus Networks Co.

\begin{abstract}
It is said that accounting is the heart of all Enterprise Resource Planning Systems (ERP). This metaphor reminds us that accounting is the center and summarization of all business information and essential to manage the business. However, this analogy is also misleading because the heart is an independent organ that pumps blood to the whole body while accounting is (1) dependent on the subsystems -auxiliary ledgers-and (2) the data it process comes from those subsystems. This paper shows how accounting data is summary data that repeats the data contained in the other subsystems; a repetition required in a manual information system but redundant and unnecessary in a computerized one. Subsequently it shows how part of the data contained in the subsystems is in itself also redundant. It concludes with an accounting data model (IAC) that provides a better data structure for computerized accounting information systems.
\end{abstract}

\section{Introduction}

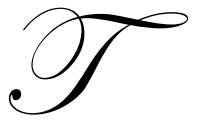

he first attempts made to computerize accounting did not question the traditional data structure of this old discipline. In fact, the first accounting databases kept the model described by Pacioli during the renaissance (Lee, Bishop, \& Parker, 1996; Macve, 1996) almost intact. That could be because a large part of that structure is theoretically grounded in concepts that are still valid today. On the other hand, another large part of that model is based in operational requirements that have lost validity with the use of computers. However, even the most well-known and up-to-date Accounting Information Systems (AIS) and Enterprise Resource Planning Systems (ERP) like SAP, ORACLE Financials, and PeopleSoft still keep some of them; the ones that are less obvious and more complex to solve and explain.

This paper highlights the parts of the traditional model that have lost validity and proposes a radically new accounting database structure that eliminates them. Keeping the status quo is the easiest, safest, and most conservative position; and conservatism is highly valued in the accounting profession. Therefore, a change to an established model used for over half a millennium is not going to be easily accepted by the community unless it is very clear and quite useful. A prior attempt was made by McCarthy (1982) with his REA model. While his model is widely accepted as accurate and interesting we found no practical implementations and while the major elements of the model are found in most ERPs they differ semantically in several critical areas because the model is too general (Weber, 1986).

To make our presentation as clear as possible we will start with a simple example that has been already corrected by most of the modern ERPs and AISs. From there, we will move towards more complex problems that are still present in most implementations.

The traditional model includes the use of Balance books where the entries are totals or subtotals of entries contained in other books (or ledgers). These Balance books are meant to avoid the need to recalculate the totals every time they are needed as long as there have been no changes to the primary data. An example of these Balance books is the Account Trial Balance. 
Figure 1 shows an example of a General Ledger and an Account Trial Balance. We can observe that the Account Trial Balance is calculated by adding together all the transactions for each account. This calculation is quick and easy with only five transactions but the General Ledger usually has many more accounts and transactions making this process long and the Account Trial Balance a necessity for practical purposes. Therefore, for practical operational purposes, the traditional accounting data structure includes not only the General Ledger but also the Account Trial Balance. The Account Trial Balance is updated every time the General Ledger is updated but only in the accounts that are affected. The update is done either with a new summarization in a new page or by altering some of the totals in an existing one.

Figure 1

\begin{tabular}{|c|c|c|c|c|c|}
\hline \multicolumn{4}{|c|}{ General Ledger } & \multicolumn{2}{|c|}{ Account Trial Balance } \\
\hline Date & Account & Number & Amount & Account & Amount \\
\hline 10-10-01 & $\mathrm{A}$ & A-001 & 15.000 & $\mathrm{~A}$ & 15.000 \\
\hline $10-10-01$ & $\mathrm{~B}$ & B-001 & 14.000 & B & 16.000 \\
\hline 10-10-01 & $\mathrm{B}$ & B-001 & 2.000 & $\mathrm{C}$ & 5.000 \\
\hline $10-10-01$ & $\mathrm{C}$ & C-001 & 1.000 & Total & 36.000 \\
\hline $10-10-01$ & $\mathrm{C}$ & C-002 & 4.000 & & \\
\hline \multicolumn{3}{|l|}{ Total } & 36.000 & & \\
\hline
\end{tabular}

Conversely, computers can calculate and recalculate quick and easily; in fact, that is one of their main advantages $(\mathrm{Oz}, 2004)$. Therefore the Account Trial Balance is an unnecessary artifact in computerized systems. Furthermore, the Account Trial Balance is not only unnecessary but its redundancy becomes a potential problem instead of an aid. It is not uncommon to find inconsistency between the primary information (General Ledger in this case) and the different totals and subtotals (Account Trial Balance). Every time a transaction is added, deleted, or adjusted the corresponding account total needs to be updated. These updates occur in different books and potentially at different times allowing for potential involuntary errors to occur. In a typical accounting the General Ledger is under constant update. Each update requires an update of the Account Trial Balance; nonetheless, this could be so burdensome that the system (or accountant) may opt to do many updates in the General Journal before finally updating the Account Trial Balance. However, the more the Account Trial Balance update is postponed the higher the possibility of making that update incorrectly. In conclusion, the Account Trial Balance should not exist in computerized systems but totals should be recalculated every time they are needed.

The Account Trial Balance is a part of the traditional accounting data structure that modern computerized systems have eliminated. Its redundancy and the potential problems it brings to accounting are so obvious that both IS professionals and accountants have agreed in eliminating them. But this is not the only redundancy present and a more methodic examination of the traditional accounting data structure is needed. Below we continue with a thorough examination of the generally accepted accounting data structure made using database theory.

\section{Redundancies}

Relational database theory states that we need to remove redundancy whenever possible (Codd, 1970). Normalization is the process that removes redundancies and hidden dependencies from a given data structure (Chen, 1976; Codd, 1971). A basic tenet of normalization is that while the final data structure may be different we are always able to recombine it in a manner that the original (and maybe redundant) representation of the data may be restored (Date, 2000).

Relational database technology includes a service known as views. A database view is an alternate form of presenting data (or a subset of the data) in a format that may be different to that in which it is physically stored (Date, 2000). Views can be used to create static (screen or printed) reports but -under certain conditions- they can also be presented on the screen as dynamic reports where the user may make changes and the system would update 
the underlying data accordingly. Views are also known as virtual tables because while they look like simple tables, the data contained on them may come from several tables or even be the result of a process (such as adding all the transactions for each account). The original data structures may be observed through views or virtual tables.

A caveat to normalization is that the views needed to present some of the original data structures may take a long time and a lot of computer power (Codd, 1970). Therefore, it is a generally accepted practice that some systems may use data structures that are not completely normalized (Date, 2000). System analysts have to choose between to evils: slow response time versus redundancies that may cause inconsistencies. Initially this problem was thought as deciding between using additional processing resources versus storage resources and since the cost of storage has dropped considerably most analyst opted towards implementing redundancies that speed up the processes. However, time has shown that the main problem with redundancies is not the need for added storage resources but the data inconsistencies that appear through time. Inconsistencies may be caused by bugs, system failures, procedural failures, or user commissions and omissions. Even in the most robust systems, users find that inconsistencies occur as they use the system. The more the system is used the more cases of inconsistencies that are found (Salim Koussa \& Ferran-Urdaneta, 2001). Most systems have modules that check inconsistencies and try to correct them; however, some inconsistencies cannot be repaired automatically since the system cannot know which of the inconsistent values is the correct one.

The prior example is a typical case of redundancy. The totals and subtotals contained in the Account Trial Balance can be calculated by adding the proper transactions contained in the General Ledger. Individual transactions and their corresponding totals are redundant; totals should not be stored. Normalizing the traditional accounting data structure eliminates the Account Trial Balance from the stored data (physically) but it still can be recreated. The first computerized AIS kept this redundancy present in the manual systems; however, current systems are implemented using more powerful hardware and they have eliminated it. Even though the underlying data structure has been altered, the user still sees all the information the traditional way. It is through views that the accounting user can still see the traditional data structure even though the data structure has been partially normalized and therefore it is stored in a different format.

\section{Redundancies in Integrated Management and Accounting Systems}

Redundancies in the traditional accounting data structure are not just exceptions. In fact, almost the entire accounting system is a pyramid made up of different levels of aggregations. They are the aggregation of the different ledgers that register the basic business transactions; like the inventory ledger and the cash ledger. These ledgers are also known as the administration ledgers. Therefore, we can say that the traditional accounting data structure is mostly redundant in respect to the administration ledgers.

\section{The Concept of Double-Entry Is Not a Redundancy}

While we just argued the pervasive existence of redundancies in the traditional accounting data structure, we will also argue that what may seem to be a redundancy, the traditional double-entry method, is not such. Doubleentry -the most basic accounting principle- seems to be a redundancy even from its own name (because of the word double); however, it is not. Double-entry means that we need to register the reception or delivery of a product or service simultaneously with the corresponding and counterbalancing delivery or reception of its value in cash or credit. There is no redundancy here because they are different but complementing elements.

Cox Benita (2003) recently wrote an article titled "Accountability lost: the rise and fall of double entry". While the title seems to forecast the demise of the double-entry method, the article in itself does not hold that hypothesis. The article presents the current problems of the datalogic accounting methods and argues that infologic methods are the way of the future accounting; however, there are solutions to those problems that do not eliminate the double-entry method. And the double-entry method is critical to assure accountability. Besides, double-entry does not hamper information usefulness which is Benita's main concern. 
The information that a product has been exchanged cannot be obtained from the information that there has been a cash exchange between those parties. Without double-entry we could record the funds received for merchandise sold without making any reference to the merchandise in itself and vice versa. In fact, that is what occurs in the two separate administration ledgers (inventory ledger and cash/bank ledger). It is the method of double-entry that forces us to relate the two records in order to keep a balance between the products delivered, the cash received, and the gain or losses that occur in each exchange. And we are forced to do it because we could not derive one from the other. What could be a redundancy is the physical storage of both transactions as separate records (McCarthy, 1979).

\section{The Physical Storage of the Separate Double-Entry Is a Redundancy}

The exchange of assets between people or organizations (or even between parts of the same organization) requires the use of documents such as receipts and reception acknowledgments. Otherwise one of the parties could always argue that they never received the assets even though they did. These documents become proof of the exchange and they are the primary source of data for both management and accounting. These documents describe the asset, identify the parties, set an agreed value, and set the date of the exchange. Accounting extracts the amounts and generates the transactions to the corresponding accounts while management uses them to manage the appropriate function. For example, with the customer's reception acknowledgment of a given merchandise, accounting deducts its cost from the inventory account, increases accounts receivable for that customers, and registers the difference between the two in the Capital Gains (or Losses) account. With the same document, management starts the accounts receivable process for that customer and the purchasing or production process for restocking the goods sold.

Proper business administration requires the use of several detailed journals to control each aspect of the process. For example, the inventory journal keeps track of purchases, sales, and transfers for each item separately. The accounts receivable journal keeps track of all receivables owned by the company and the corresponding payments made by each customer without describing how the receivables were caused or where the funds credited were applied. The banking journal lists the cash flows to each bank account but it does not fully explain the origin or application of the funds. On the other hand, accounting registers all the transactions mentioned earlier (in a detailed or summary form at the accountant's choice) but making sure to explain where incoming funds are placed or where outgoing funds come from. In other words, the sum of all the credits is equal to the sum of all the debits. This way accounting assures the proper interrelationship between auxiliary journals which is what is called double-entry bookkeeping. From this point of view, accounting seems to be indispensable and related but separate from the auxiliary ledgers. However, this duplication is a necessary artifact of manual systems only.

It is very difficult for an individual to assure that all that is registered in one journal is properly counter registered in another journal since all of these journals are physically separate. This is even more difficult when more than two ledgers are involved (for example a sale that is partially paid in cash and partially paid in credit affects the bank, account receivable, inventory, and sales journals). Registering and duplicating all of these transactions in a single journal (or general journal) brings them together in a way that the accountant can then make sure that the debits are equal to the credits. However, this physical duplication that brings them together is unnecessary in a computerized system. Computerized database systems can present the General Ledger as a "view" of the auxiliary ledgers making the physical storage of the General Ledger an unnecessary redundancy.

The redundancy between the accounting and the administration information is only one of several redundancies present in the data structure of current AISs and ERPs. These modern systems also hold similar redundancies between the journals of different sub-modules. Following parts will discuss the two most common inter-auxiliary journal redundancies. 


\section{Redundancy between Invoice Header and Customer/Supplier Statement of Account}

This redundancy occurs in modular designs where the invoicing module (sales) stores the data separately from that of the customer or the supplier module (accounts receivable or accounts payable respectively). To explain this redundancy let us use the invoice shown in figure 2.

Figure 2

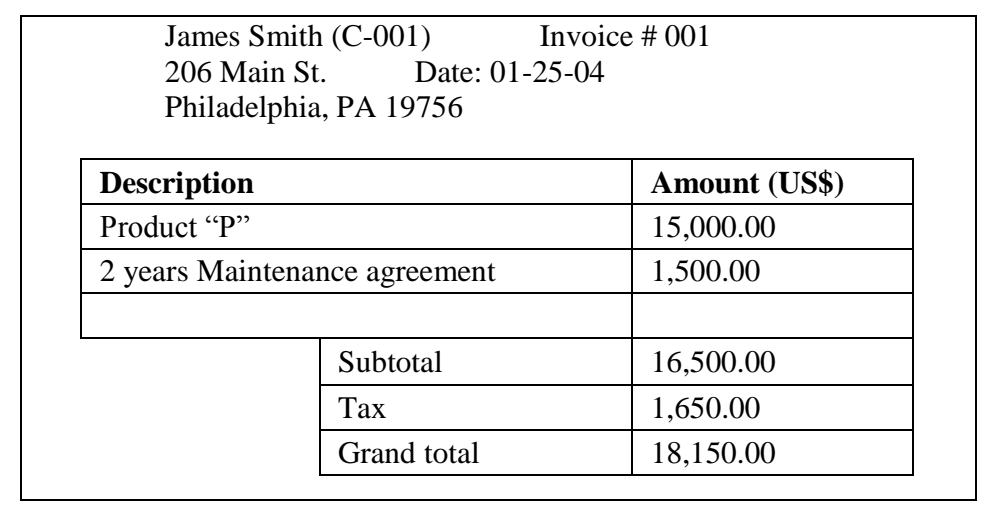

The invoicing module data structure would be made up of two tables: invoice header and invoice detail. Invoice detail would include the invoice number (to establish the relationship with the invoice header), the item description (or an ID or SKU that relates it to yet another table) and the amount (many others could also be included like quantity, unit price, and discount but we omit them for sake of simplicity). The invoice header would include the invoice number, date, customer ID, and maybe a few others. It could also include subtotal, tax, and grand total although these are an added redundancy. In addition, the accounts receivable module would have a table with the document number, date, type (invoice or credit for example), the customer ID, the invoice grand total, a description, and perhaps a few others. Figure 3 shows the data structure in table notation.

\section{Figure 3}

InvoiceHeader(InvNum, Date, CustID, ...)

InvoiceDetail(DetailID, InvNum, ItemDesc, Amount, ...)

Receivable(DocNum, Date, Type, CustID, Descript, Amount, ...)

BankDetail(AccNum, Date, Type, TransID, CustID, Descrip, Amount, ...)

The redundancy is obvious. Most (if not all) of the Invoice Header information is also in Receivable. Furthermore, there is a one-to-one relationship between these two tables.

\section{Redundancy between Accounts Receivable / Accounts Payable and Cash/Bank Ledger}

This redundancy occurs in modular designs where the customer or the supplier module (accounts receivable or accounts payable respectively) stores the data separately from that of the Cash and Bank module. To explain this redundancy let us use the invoice shown in figure 4.

In this case the receivables table would have a second record that corresponds to the US $\$ 14,000.00$ paid in cash and the bank transaction table would also be used. Figure 5 shows the data in the receivables table as well as the data in the bank transaction table. 
There is clearly a redundancy between the date and amount in DocNum Cb-001 in the Receivables table and the date and amount in TransID 0102991 of the Bank journal table.

Figure 4

\begin{tabular}{|c|c|c|}
\hline $\begin{array}{l}\text { James Smith } \\
206 \text { Main St. } \\
\text { Philadelphia, }\end{array}$ & $\begin{array}{l}\text {-001) } \\
\text { Date: } 01-2 \\
19756\end{array}$ & $\begin{array}{l}\text { James Smith (C-001) Invoice \# } 001 \\
206 \text { Main St. } \quad \text { Date: } 01-25-04\end{array}$ \\
\hline \multicolumn{2}{|l|}{ Description } & Amount (US\$) \\
\hline \multicolumn{2}{|l|}{ Product "P" } & $15,000.00$ \\
\hline \multicolumn{2}{|c|}{2 years Maintenance agreement } & $1,500.00$ \\
\hline & Subtotal & $16,500.00$ \\
\hline & Tax & $1,650.00$ \\
\hline & Grand total & $18,150.00$ \\
\hline & Cash & $14,000.00$ \\
\hline & Amount due & $4,150.00$ \\
\hline
\end{tabular}

Figure 5

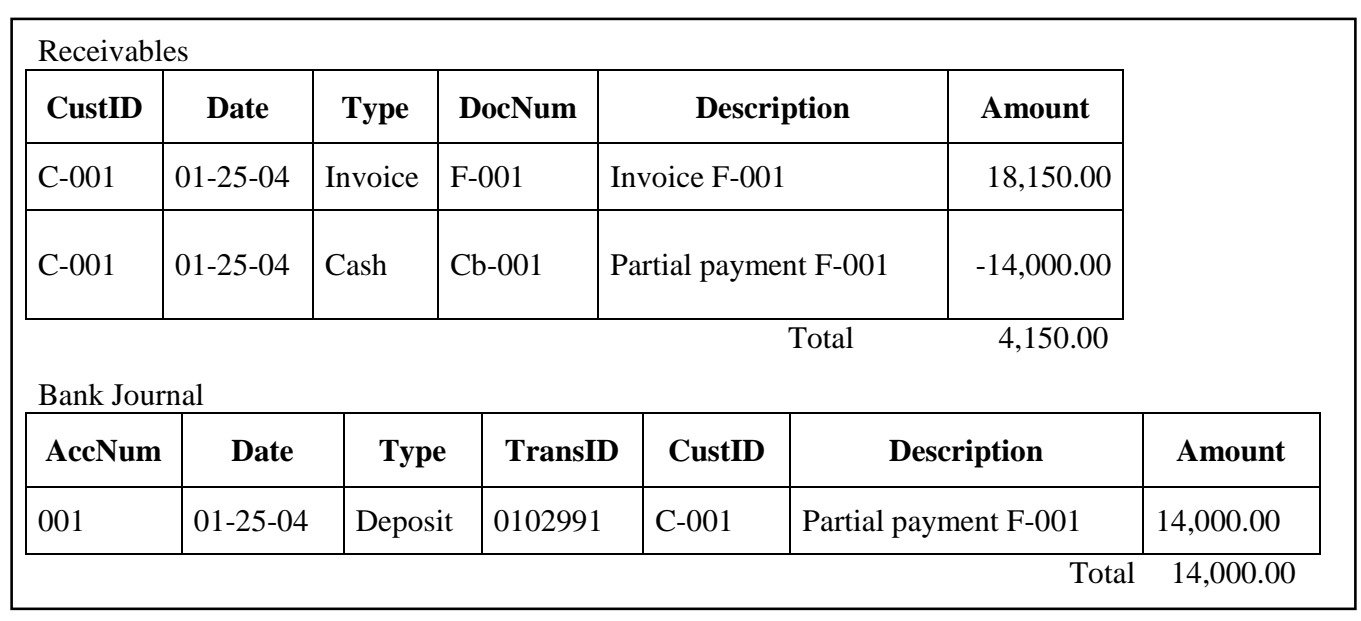

\section{The IAC Model}

In this section we describe a model with an alternate accounting data structure that radically eliminates the most pervasive and potentially inconsistent redundancies of the traditional administrative-accounting model. The model is called IAC and makes up the data structure of the SecureAccounting system (both property of Cautus Network Corporation, Miami, FL.). The system (and in consequence the underlying data structure -IAC-) has been in place and under intensive use in over fifty companies in the past two years. The name IAC comes from the initials of Items, Agents, and Cash.

\section{Items, Agents, and Cash}

The traditional accounting model contains five types of entities. They are Assets, Liabilities, Capital, Revenues, and Expenses. The three main entities of IAC, the model we propose, are Items, Agents, and Cash. They 
reclassify and consolidate all the entities of the traditional model. While the IAC classification may be more or less intuitive than the traditional one, it is a more normalized data structure that in turn eliminates many of the redundancies present in the traditional model.

The traditional model subdivides the main accounts (entities) in sub-accounts. Many of the sub-accounts are kept in separate books because of their nature and structure. Furthermore, some sub-accounts that originate from different main accounts are also kept separate even though their structure is very similar if not the same. For example, Accounts receivable is an Asset and Accounts payable is a Liability; they are kept separate although their structure is almost the same. There probably is a third group of sub-accounts called Employees (used for payroll purposes at a minimum), which is also kept separately. The IAC model consolidates customers, suppliers, employees, and stockholders into one entity: Agents. In general, Agents are all people and institutions that exchange cash, goods, and/or services with the company. They all have many attributes in common (like name, address, and balance) but what is even more important, they all have the same type of database relationships with the other two main entities (Items and Cash).

The entity Item consolidates Fixed Assets, Inventory and all the goods and services that the company buys or sells. They are kept in separate books although they have many attributes in common (like name, description, measurement unit, quantity, cost, and price). Furthermore, they all have the same type of database relationships with the other two main entities (Agents and Cash). of money.

Finally the entity Cash consolidates bank accounts, petty cash, and any other account that reflects the flow

Administrative and accounting systems register the economic activity of a company. In general, this economic activity is made up of the exchange of goods or services for money (or in certain cases for other goods or services). The Agent entity responds to the question of "Who" (surrenders or receives the goods or services); Item responds to the question of "What" (goods or services); and Cash to the "How much" (money it is given or received in exchange for the goods or services). This way the three IAC entities cover all the entities involved in economic activity and therefore consolidates all the accounts of an administrative or accounting system.

However, consolidating all sub-accounts into three main entities is a necessary but not sufficient condition to eliminate the redundancies present in the traditional model. Further work and explanation is needed to eradicate them.

The following three sections mirror (but in reverse order) the last three sections of the previous chapter. They explain how IAC eliminates each of those redundancies.

\section{Eliminating the Accounts Receivable / Accounts Payable - Cash/Bank Ledger Redundancy}

Previously we showed -in Figure 5- how the date and amount in DocNum Cb-001 in the Accounts Receivables table and the date and amount in TransID 0102991 of the Cash/Bank Ledger table were redundant (as well as some other attributes). To solve this redundancy it is enough to add the unique attributes (like bank account, type, number, and transactionID) of the Cash/Bank Ledger table to the Accounts Receivable table and eliminate the whole Cash/Bank Ledger table.

Now then, we have created a single table (or entity) called AgentTransactions which is the fusion of Account Receivable transactions and the Cash/Bank ledger. Therefore, the AgentTransactions table of the IAC model replaces the two above mentioned tables of the traditional model. Figure 6 shows the new table and its data based on our last example.

Total accounts receivable is calculated by adding up all the transactions. Total cash is calculated by adding up all the transactions where AccNum is not null and then reversing the sign of the total. 
Note that this radical solution eliminates the Cash/Bank ledger as a physical table and calculates it based on views of the AgentTransaction table. Therefore, while Cash is one of the three main entities of the IAC model it exists only in a logical form; not as a physical table.

Figure 6

\begin{tabular}{|c|c|c|c|c|c|c|c|c|}
\hline CustID & Date & Type & DocNum & Description & AccNum & Type & TransID & Amount \\
\hline C-001 & $01-25-04$ & Invoice & F-001 & Invoice F-001 & & & & $18,150.00$ \\
\hline $\mathrm{C}-001$ & $01-25-04$ & Cash & $\mathrm{Cb}-001$ & Partial payment F-001 & 001 & Deposit & 0102991 & $-14,000.00$ \\
\hline & & & & & \multicolumn{3}{|c|}{ Total Accounts Receivable } & $4,150.00$ \\
\hline & & & & & \multicolumn{3}{|l|}{ Total Cash } & $14,000.00$ \\
\hline
\end{tabular}

\section{Eliminating the Invoice Header - Customer/Supplier Statement of Account Redundancy}

In a previous section we described two redundancies: (1) the invoice total can be calculated by adding its details; however, systems often physically record the total in the invoice header too, (2) most of the data included in the invoice header is also found in the corresponding account receivable transaction.

To solve the redundancy between the invoice header and the receivables detail we eliminate the invoice header (or any similar header table like the purchase order header table) from the database design. We selected to eliminate the invoice header and not the receivables transaction since all invoice headers will be reflected in the receivables transactions while not all the receivable transactions will be reflected in the invoice headers (all invoices generate a receivable -even a "dummy" or "hidden" receivable with a cero amount if fully paid in cash- but not all the receivables come from invoices).

In the IAC data structure the invoice information is stored partially under Agents and partially under Items. Under Agents we have the invoice header information and under Item we have the invoice detail information. The header describes the party involved, the date, and the conditions. The detail describes the what, the how many, and the how much is being transacted.

The conventional solution to solving the redundancy of physically storing a total is to calculate it every time is needed. However, we (like most major software developers) have chosen -for the sake of performance- to keep the redundancy and store the total, at least for the first practical implementation of the IAC model (SecureAccounting). Showing an invoice's total is a very frequently used option on administrative / accounting systems; therefore, calculating it every time instead of reading the result from a physical location substantially increases response time. This exception is a process of denormalization based on operating requirements.

Yet another reason not to eliminate this redundancy is that the receivable detail table includes records that come from invoices but also records that are later payments to the account. When the record originates from an invoice then the amount is the sum of the items in the invoice (clearly a redundancy); but when it represents later payments it cannot be calculated, it has a meaning of its own, and it is not a redundancy. We could still place a null value for the former and a number value for the latter to avoid the redundancy but this would make the addition of records in this table a complex (and slow) process that would not make use of standard (and quick) summation features found in current database management systems.

\section{Eliminating the Redundancy between the Accounting and the Subsidiary Ledgers}

As discussed earlier the accounting ledgers are meant for assuring the use of the double-entry method. They are not needed for making the double-entry, only to guarantee that the double-entry was made properly. For example, in the case of a sale, the accounting ledger physically brings together the total charged to a customer with the total subtracted from inventory and the difference applied to gain or losses. While these three are already stored 
in separate subsidiary ledgers, the traditional model -as well as most current ERP systems- still stores them in a separate set of accounting ledgers -or tables.

Under the IAC model the accounting ledgers are virtual ledgers. The double-entry is assured by bringing the records together in a logical -not a physical- manner. The records are stored as Item, Cash, and Agents -as the primary data- and the general journal entry is a simple view (or virtual table) extracted from them. In this way, IAC radically cuts out the redundancy between the Accounting and the Subsidiary Ledgers.

Even though all accounting entries should come from the subsidiary ledgers, many accountants still either modify the entry directly in the accounting ledger and not in the subsidiary ledger, or create it in accounting without registering them in the subsidiary ledger. A system that would not allow them to directly work with the accounting ledger would not be welcome. However, one that would allow them to do so and still keep the consistency with the subsidiary ledgers would be of a great help.

The IAC model allows direct manipulation of General Ledger entries without losing consistency with the subsidiary ledgers. This can be easily done with simple and consistent DBMS procedures because all the information is stored in two physical tables (Agent and Item) and one logical table (Cash). In turn this also allows for fast response time when generating or regenerating views.

Converting the accounting ledgers into simple views provides increased data integrity while not slowing down reports significantly. Generating a balance sheet or an income and losses statement is not appreciably faster under the traditional model than under the IAC model. Furthermore, a test of the integrity between subsidiary ledgers and the accounting ledger is not needed under the IAC model because they are one and the same data. Moreover, a fundamental integrity test (ex. check if the fundamental accounting equation holds) under the IAC model is not just about accounting but a comprehensive check of the entire data (accounting and subsidiary ledgers). And it is done so fast that it can be included at the end of each database update without apparent performance degradation.

\section{Advantages for the Application Programmer}

As previously stated, the traditional accounting and administrative data structure responds to the need to optimize response times on paper and pencil (manual) systems; however, what is optimal for manual system is not always such for computerized ones. Nonetheless, the traditional data structure has been kept and the ledgers are faithfully reflected in the database designs of most computerized accounting and administrative systems. In other words, the needed reengineering of the database structure never took place. Therefore, the reorganization task has been pushed to a different area: application programming, which was already overworked and is not the optimal person to solve this problem. Applications programmer not only have to write the complex code for forms, reports, and data processing, but also must temporarily reorganize the database twice: once to take advantage of the computerized methods and a second time to display the information the traditional way. Under the IAC model this temporary database reorganization is unnecessary freeing the application programmer to do the task that s/he was trained to do.

An example of how the IAC model simplifies the job of the application programmer is the existence of a simple (and quick) DBMS function that allows verification of the basic accounting equation from within a single administrative module. This way, any transaction that affects accounting may be validated directly from the application modules even if they are disconnected from the main accounting system. This validation is impossible to do under the traditional DB model for at least two reasons: First, the information is scattered through many different tables and therefore such test would take a long time to process; and second, such a validation would never be definitive because the accounting transaction is not created immediately and requires a series of intermediate steps where inconsistencies may later appear.

Another example is the facility to develop summary and high level reports. Under both the traditional model and the IAC model it is fairly easy to develop detailed or low level reports. Under IAC we scan the two major 
tables applying filters to select the appropriate transactions and then apply sorting and presentation algorithms. In a similar way, under the traditional model, we do the same scanning of the tables (which would be of smaller size) and apply the same sorting and presentation algorithms. Summary reports under the IAC model are developed the same way as detailed ones; however, under the traditional model they are far more complicated. The programmer needs to scan several tables, each with a different structure, and create a temporary table that includes all the selected transactions to then apply the necessary sorting and presentation algorithms.

In conclusion, under the IAC model, the application programmer concentrates in programming forms, reports, and processing data and the DBMS takes care of most validations and search processes. Under the traditional model the application programmer needs to perform tasks that are not normally part of his/her responsibility incurring in the bad practice of using resources that are not meant nor optimal for such tasks.

\section{The Fundamental Accounting Equation under IAC}

Assets are the resources that a company owns. When a company is started, the only assets it holds are those that stockholders provide as their initial investment. The company may also acquire additional assets by incurring in debt. Therefore, total assets will be equal to the sum of the assets provided by the stockholders plus the sum of the assets provided by the creditors. This is what is called the fundamental accounting equation and it is written as follows:

\section{Assets $=$ Liabilities + Capital}

The fundamental accounting equation must be held true at all times. Each side of the equation is calculated separately and compared to check that the equation holds. Therefore if we add all the assets we will get the same number than if we add together all the debt provided by creditors and stockholder plus the equity.

As the accounting cycle develops, two more entities are used: Revenues and Expenses. At any time we may add all the Revenues and subtract all the Expenses and we would get the operating result which is part of the Capital. Summarizing: the traditional accounting model contains five types of entities: Assets, Liabilities, Capital, Revenues, and Expenses. Revenues and Expenses are operating entities that show the results of the company's activity during a given period; they are cleared at the end of the period and the difference between them is added (or subtracted) from the Capital account.

The traditional accounting model classifies information based on ownership (the company owns the assets, creditors own the liabilities, and stockholders own the capital). On the other hand the IAC accounting model classifies information based on data structure similarities which not only simplifies the use of information technology to process the data but also helps in eliminating redundancies. Moreover, although the classification is different the model still allows easy testing of the fundamental accounting equation; in fact, it makes the process faster and easier.

We can apply the logic of the fundamental equation in different terms. For example, a company acquires assets in exchange for cash or debt from stockholders or third parties. It can also sell goods (or services) in exchange for cash or loans given to third parties. Therefore, following a similar method to the one applied earlier to obtain the fundamental accounting equation we get that:

\section{Account of Goods $($ Items $)=$ Accounts of People $($ Agents $)+$ Accounts of Money $($ Cash $)$}

where each side of the equation is calculated independently of the other as required by the double-entry bookkeeping method.

The process required under the IAC model to corroborate the fundamental equation is very simple making it even more reliable than the traditional one. All we have to verify is that: 


\section{$\sum$ Items(Amount) $=\sum$ Agents(Amount) $+\sum$ Cash(Amount)}

and since all the data is in only two tables very little programming is needed.

To guarantee that the fundamental equation holds for the whole, we need to assure that it holds for each part. In other words, every time we register an economic event (i.e. purchase, sale, etc) we also need to check that the set of transactions recorded maintain the equation. However, this is quite easy and intuitive. Every merchandise or cash received or surrendered comes or goes to a person or institution (even if it is to the same company or unit or department). Therefore the amount of every transaction record in Agents will be the same to the related transaction in Cash and Items. In more technical terms, let AgentTransID be the attribute in Cash and/or Items that contains the ID of the related transaction in Agents (in other words, AgentTransID is the foreign key in Cash and Items that relates the records to Agents whose primary key is ID). We then have that:

\section{$\sum$ Agent(ID, Amount) $=\sum$ Item(AgentTransID $=$ ID, Amount $)+\sum$ Cash(AgentTransID $=$ ID, Amount $)$}

\section{Conclusions}

It is debatable which classification (the traditional or IAC) is more intuitive; however we have shown that both are equally effective to control the required balance in bookkeeping (verifying the fundamental accounting equation) and IAC is easier to implement in computerized information systems. IAC also eliminates most of the redundancies present in the traditional classification. Furthermore, we can use the IAC data structure for physical storage and still use the traditional data structure for data presentation and manipulation at the user level. Finally, IAC is not simply a theoretical model looking for an application but a theoretically grounded model that has been tested and is implemented in accounting software that has been in use in over fifty companies for the past three years.

\section{Acknowledgments}

We thank Cautus Network Corporation of Miami, FL for their permission to publish this paper on its radically new accounting data model.

\section{References}

1. Benita, C. (2003). Accountability lost: The rise and fall of double entry. Omega The International Journal of Management Science, 31((2003)), $303-310$.

2. Chen, P. P.-S. (1976). The Entity-Relationship Model - Toward a Unified View of Data. ACM Transactions on Database Systems, 1(1), 9-36.

3. Codd, E. F. (1970). A Relational Model of Data for Large Shared Data Banks. Communications of the ACM, 13(6), 377-387.

4. $\quad$ Codd, E. F. (1971). Normalized data base structure: A brief tutorial. Paper presented at the ACM-SIGFIDET 1971 Workshop: data processing, access and control, San Diego, CA.

5. Date, C. J. (2000). An introduction to database systems (7th ed.). Reading, Mass.: Addison-Wesley.

6. Lee, T. A., Bishop, A. C., \& Parker, R. H. (1996). Accounting history from the Renaissance to the present: A remembrance of Luca Pacioli (New works in accounting history). New York: Garland Pub.

7. Macve, R. H. (1996). Pacioli's Legacy. In T. A. Lee, A. C. Bishop \& R. H. Parker (Eds.), Accounting history from the Renaissance to the present: a remembrance of Luca Pacioli (New works in accounting history) (pp. xxiv, 290). New York: Garland Pub.

8. $\quad$ McCarthy, W. E. (1979). An Entity-Relationship View of Accounting Models. The Accounting Review, $54(4), 667$.

9. McCarthy, W. E. (1982). The REA Accounting Model: A Generalized Framework for Accounting Systems in a Shared Data Environment. The Accounting Review, 57(3), 554.

10. Oz, E. (2004). Management Information Systems (4th ed.). Boston, MA: Course Technology.

11. Salim Koussa, R., \& Ferran-Urdaneta, C. (2001). Neither a perpetuum mobile nor a perfect software: Sincerity in the relationship between the manufacturer and the client with respect to software defects. Paper presented at the 5th World Multi-Conference on Systemics, Cybernetics and Informatics (SCI'01/ISAS'01), Orlando, FL.

12. Weber, R. (1986). Data Models Research in Accounting: An Evaluation of Wholesale Distribution Software. The Accounting Review, 61(3), 498-518. 
$\underline{\text { Notes }}$ 